\title{
Planetary portraits
}

The list of known

planets outside our Solar System grows by the week. Thanks to new technologies, a host of pictures of these worlds could soon be available. Tony Reichhardt investigates.

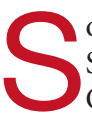
oon after the first planet orbiting a Sun-like star was discovered, Dan Goldin, then administrator of NASA, issued a challenge. At a meeting of the American Astronomical Society (AAS) in San Antonio, Texas, in January 1996, he called on the assembled scientists to find a way, within 25 years, to take a picture of an Earth-sized planet orbiting a nearby star with a resolution good enough to see oceans, clouds, continents and mountain ranges. "And if that doesn't get your heart pumping," Goldin added, "I don't know what will."

Hearts may have pumped, but eyes also rolled. Surely Goldin understood the daunting technical problems. An Earth-sized planet orbiting a nearby star like our Sun would be lost in the glare from its parent, which would shine 10 billion times more brightly. Even a Jupiter-sized planet would be a billion times dimmer than its stellar companion.

But state-of-the-art space cameras can handle contrast levels of only around a million to one. Images of oceans and mountains were out of the question, and many astronomers doubted whether even crude imaging of extrasolar planets was feasible in the forseeable future.

Fast forward six years to last month's annual AAS gathering, held in Washington. Extrasolar planet-hunting is now big business. Seventy-five have been discovered, and the list is growing by the week. Once relegated to back-room sessions, discussions of extrasolar planets filled the largest conference halls. And thanks to new concepts in telescope design, there was serious talk of moderately priced space missions that could return visible-light pictures of planets within five or six years. Goldin's pictures of alien
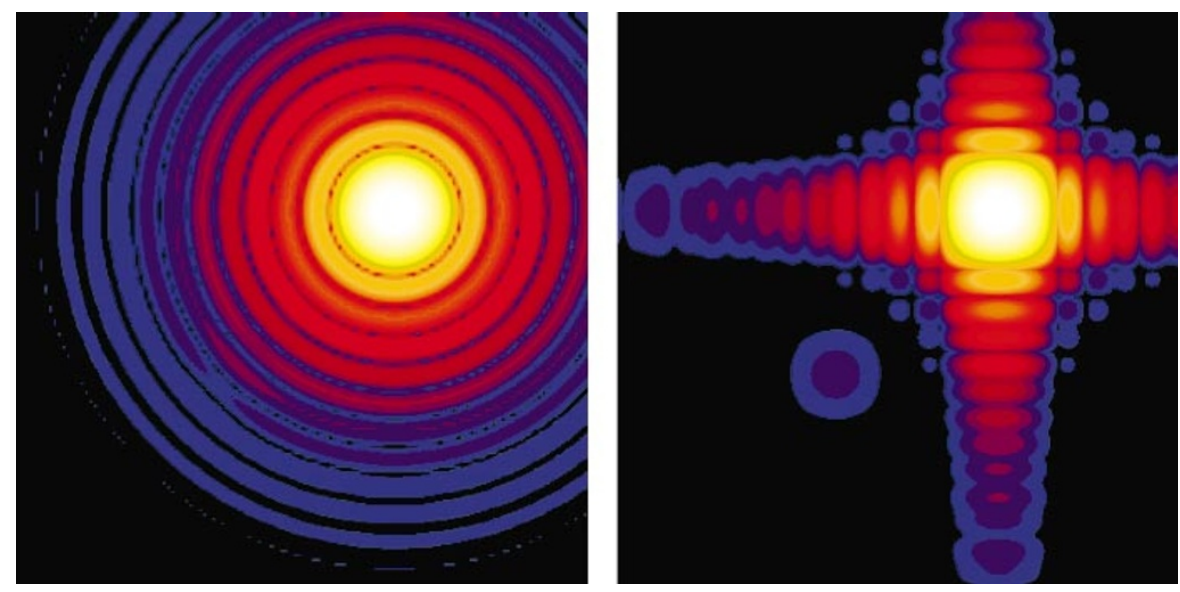

Brave new worlds: a brown dwarf (bottom left, top picture) next to a star. Simulations of new telescope apertures (bottom pictures) show how dimmer objects could be imaged. A square aperture (right) reveals a planet that is hidden by diffracted light when a circular aperture is used (left).

oceans remain mission impossible for now, but the first visible-light images of Jupitersized planets may be taken sooner than even optimists had thought.

\section{Cutting the odds}

Ground-based telescopes can already photograph brown dwarfs - objects that are intermediate in size between planets and stars using infrared light, in which the brightness difference between the dwarf and the star it orbits is less pronounced. At last month's AAS meeting, Michael Liu of the University of Hawaii presented pictures (see above) of such an object orbiting 14 astronomical units from its star ( $1 \mathrm{AU}$ is the distance from the Earth to the Sun).

But capturing visible-light images of Jupiter-sized planets will require new orbiting telescopes, as well as advances in imaging technology. Such improvements are already in the offing, thanks to developments in coronagraphy - the technology of blocking unwanted light — and the techniques used to manufacture telescope mirrors.

The conventional approach to masking starlight is to place an opaque disk in the centre of the telescope's circular aperture, or to 'fog' selected areas of the telescope's mirror so that it does not reflect light from the star. But light that is diffracted by the edge of the aperture, along with light scattered by imperfections in the mirror, creeps in, creating a background glow that can obscure planets.

Rather than using a light-blocking disk, Peter Nisenson of the Harvard-Smithsonian Center for Astrophysics in Cambridge, Massachusetts, and Costas Papaliolios of Harvard University, hope to solve the problem by using a square aperture (see 

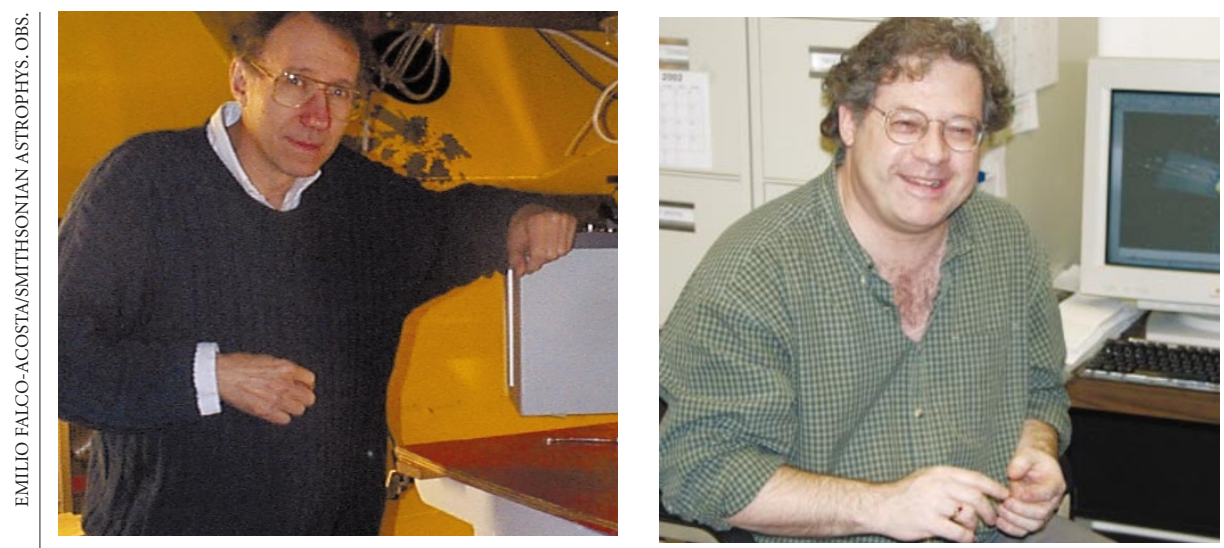

Giant hunters: Peter Nisenson (left) and Gary Melnick hope to image Jupiter-sized planets.

P. Nisenson \& C. Papaliolios Astrophys. J. 548, L201-L205; 2001). In contrast to the ring-like diffraction pattern created by circular apertures, their square aperture produces a cross-shaped pattern which can be rotated until the planet falls in one of the dark areas outside the bright cross (see left).

A modified version of this system is at the heart of the Extra-Solar Planet Imager (ESPI), a space telescope proposed to NASA by Gary Melnick, also at the Harvard-Smithsonian Center for Astrophysics. Launched from the space shuttle into Earth orbit, it would use a 1.5-metre-square aperture to take long exposures of 160 or more stars that are similar in age to our Sun and situated within about 50 light years of Earth. Melnick says the system could achieve the contrast necessary to image Jupiter-sized planets at 5 $\mathrm{AU}$ from their parent stars, which is Jupiter's distance from our Sun. For a handful of very close stars, pictures of planets that are only a few times more massive than Earth and only 1 AU from their stars might be possible.

But the benefits of the square aperture are not free. Fine-tuning the placement of diffracted light from the central star makes the whole system much more sensitive to imperfections in the optics. "Any stray light that can sneak into that dark region can fool you," says David Black of the Lunar and Planetary Institute in Houston, Texas, a veteran of the extrasolar-planet field.

So the ESPI project is counting on a new generation of mirrors, built with unprecedented smoothness and accuracy of shape, that have been developed for computer-chip manufacturers. In the quest to create eversmaller microprocessors, the semiconductor industry has advanced the technology it uses to etch circuits onto wafers of silicon. Known as extreme-ultraviolet lithography, the new technique uses ultraviolet light to create circuit components that are less than 100 nanometres across. Optics companies have developed methods for making new and smoother mirrors to focus the beams of ultraviolet light. Melnick hopes that these techniques, which involve using ion beams to strip away layers of atoms one at a time, will soon be applied to telescope mirrors.

So do advocates of the Jovian Planet Finder (JPF), another proposed planetimaging mission. Principal investigator Mark Clampin of the Space Telescope Science Institute in Baltimore, Maryland, sees mirror imperfections as the biggest challenge. Rather than new apertures, he will use a series of super-smooth mirrors to search for Jupiter-sized planets around 50 stars, smaller Saturn-sized planets around 34 stars, and brown dwarfs around 2,000 stars. The JPF's planned vantage point is unorthodox: it would cling to the outside of the International Space Station.

Like the ESPI, the JPF has been proposed to NASA as a candidate for a Medium-Class Explorer (MIDEX) mission to be launched in either 2007 or 2008. NASA will select several MIDEX mission concepts for further study in April, and two winners will be selected by the end of the year. If chosen, the projects would receive a budget of up to $\$ 198$ million.

\section{Computer corrections}

A third mission concept, Eclipse, was presented at the AAS meeting by John Trauger of the Jet Propulsion Laboratory in Pasadena, California. Eclipse would use fairly conventional coronagraphy, and would not rely on the new super-smooth mirrors. Instead, it would use a computer to adjust the shape of a deformable mirror, and so continually correct for inaccuracies in the image caused by imperfections in the mirror's surface. Proposed to NASA's Discovery Program, which allows for missions costing up to $\$ 300$ million, Eclipse would be able to detect Jupiter-sized planets within 5 AU of bright nearby stars, and could even see Uranus-sized planets in a few systems.

If these missions appeal to NASA's review panels, they could serve as warm-ups to the Terrestrial Planet Finder (TPF), a proposed NASA mission planned for a 2014 launch and expected to cost between $\$ 1.3$ billion and $\$ 2.8$ billion. The TPF will be sensitive enough to image Earth-sized planets, and will also study their spectra, searching for signs of life such as the signature of molecular oxygen.
Four groups are currently in contention to build the TPF. The field will be narrowed to two this spring, with the design to be finalized in 2006 - all subject to the vagaries of NASA funding and the possibility of a merger with the European Space Agency's Darwin project, which has broadly similar aims.

Designs for the TPF and Darwin have focused on the use of nulling interferometers that operate in the infrared spectrum. These devices combine images from several telescopes. If the distances between them are chosen correctly, the starlight that reaches the different telescopes will be out of phase and will cancel out when the images are combined, leaving only the faint glow from a planet.

But working with infrared wavelengths has a drawback: a larger telescope aperture is required to achieve the same resolution that smaller apertures can obtain when working with visible light. So designs for the TPF and Darwin have called for an array of telescopes, either flying in formation or yoked to a boomlike structure as much as 100 metres long. Cryogenic cooling might also be required to cool the detector to prevent images being swamped by infrared radiation from the detector itself, a detail that further complicates the design. And some of the most interesting spectral signs of life lie in the visible, rather than infrared, spectrum.

With new ideas for imaging planets in visible light now emerging, opinions on the TPF have begun to change. Two of the four teams - those based at Ball Aerospace and Technologies in Boulder, Colorado, and BoeingSVS in Albuquerque, New Mexico - are now considering TPFs that would operate in the visible spectrum. "The whole game is now a bit more open," says Black.

With a range of planet imagers on the drawing-board, and a revamped TPF under consideration, the next few years should be a busy time for planet-hunters. Goldin's extrasolar landscapes may yet be a few decades away, but his challenge is starting to look a little more realistic.

Tony Reichhardt is a contributing correspondent for Nature. AAS Washington meeting abstracts www.aas.org List of known extra-solar planets www.obspm.fr/planets Terrestrial Planet Finder tpf.jpl.nasa.gov

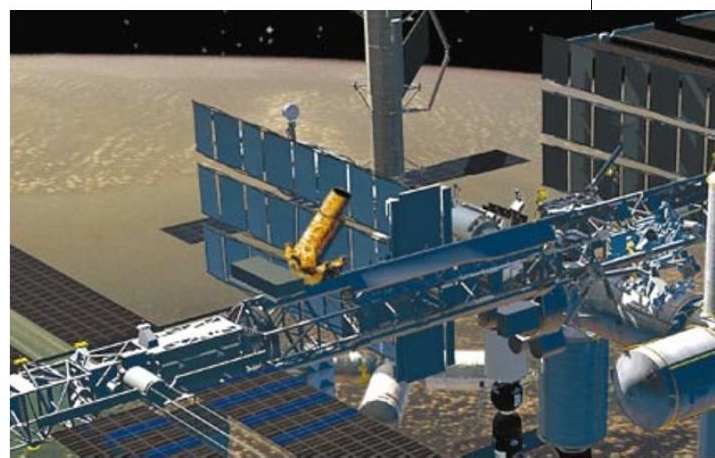

Cling on: a mock-up of the Jovian Planet Finder attached to the International Space Station. 